\title{
HARMONIC MORPHISMS APPLIED TO CLASSICAL POTENTIAL THEORY
}

\author{
BENT FUGLEDE
}

\begin{abstract}
It is shown that if $\varphi$ denotes a harmonic morphism of type Bl between suitable Brelot harmonic spaces $X$ and $Y$, then a function $f$, defined on an open set $V \subset Y$, is superharmonic if and only if $f \circ \varphi$ is superharmonic on $\varphi^{-1}(V) \subset X$. The "only if" part is due to Constantinescu and Cornea, with $\varphi$ denoting any harmonic morphism between two Brelot spaces. A similar result is obtained for finely superharmonic functions defined on finely open sets. These results apply, for example, to the case where $\varphi$ is the projection from $\mathbb{R}^{N}$ to $\mathbb{R}^{n}(N>n \geq 1)$ or where $\varphi$ is the radial projection from $\mathbb{R}^{N} \backslash\{0\}$ to the unit sphere in $\mathbb{R}^{\bar{N}}(N \geq 2)$.
\end{abstract}

\section{$\S 0$. Introduction}

Aside from holomorphic maps between Riemann surfaces, harmonic morphisms can be traced back to Jacobi [Jac] in 1848 for maps between $\mathbb{R}^{3}$ and $\mathbb{C}$. The first systematic study of harmonic morphisms (then termed harmonic maps) was made by Constantinescu and Cornea [CC2, Section 3] in the very general potential theoretic setting of continuous maps between harmonic spaces in the sense of Brelot, such that the pullback of any harmonic function in $Y$ is harmonic in $X$ (see [Br, Part IV], [CC3] for details concerning Brelot harmonic spaces). A particular case is that of harmonic morphisms between Riemannian manifolds; they were characterized as harmonic maps (in the sense of differential geometry) which, moreover, had a certain property of conformality (see [F4], [Ish]). For a comprehensive account of the theory of harmonic morphisms between Riemannian manifolds, we refer to the excellent monograph by Baird and Wood [BW].

In Section 1 of this paper, we establish further properties of harmonic morphisms between Brelot spaces (especially maps of type $\mathrm{Bl}$ in the sense of [CC2]), generalizing analytic functions represented by a Blaschke product

Received February 3, 2010. Revised September 2, 2010. Accepted September 13, 2010. 2010 Mathematics Subject Classification. 31B05, 31C05, 31C12, 31C40 
(see also $[\mathrm{Hs}]$ ). Thus we establish reverse implications to some key definitions and results of [CC2, Section 3] and [F3]. The first study similar in scope to this one was made by Hansen [Ha], who allowed the more general harmonic spaces introduced by Bauer $[\mathrm{Ba}]$ and Constantinescu and Cornea [CC3], but imposed a stronger hypothesis than that of type Bl (see also [BH, Section V.8]).

In Section 2, we study the particular harmonic morphisms given by projection from $\mathbb{R}^{N}$ to $\mathbb{R}^{n}(N>n \geq 1)$ and by radial projection from $\mathbb{R}^{N} \backslash\{0\}$ to the unit sphere $\mathbb{S}^{N-1}$ in $\mathbb{R}^{N}(N \geq 2)$. We thereby recover known results by Deny and Lelong [DL], Hansen [Ha], and Gardiner [G1], [G2], and we add a result on finely superharmonic functions.

Finally, in Section 3, we extend the results of Section 1 to so-called finely harmonic morphisms, whose domain of definition is a finely open subset $X$ of an ambient Brelot space, as described in [F3].

\section{$\S 1$. Harmonic morphisms}

Following Constantinescu and Cornea [CC2], we will consider harmonic morphisms $\varphi: X \rightarrow Y$ between Brelot spaces $X$ and $Y$ (see [Br, Part IV], [CC3, Section 3.1], [La1]; for harmonic morphisms between more general harmonic spaces, see [Me], [La2]; for harmonic morphisms in nonlinear potential theory, see $[\mathrm{HKM}])$.

Definition 1 ([CC2, Section 3]). A continuous map $\varphi: X \rightarrow Y$ between Brelot spaces $X$ and $Y$ is called a harmonic morphism if, for any open subset $V$ of $Y$ and any harmonic function $f$ on $V, f \circ \varphi$ is harmonic on $\varphi^{-1}(V)$ (when nonempty).

It would amount to the same replacing harmonic (twice) by hyperharmonic or by hypoharmonic (see [CC2, Theorem 3.1]).

If $X$ is connected, if $\varphi: X \rightarrow Y$ is a nonconstant harmonic morphism, and if $f$ is a superharmonic (resp., subharmonic) function on an open set $V \subset Y$, then $f \circ \varphi$ is superharmonic (resp., subharmonic) on $\varphi^{-1}(V)$ (when nonempty) (see [CC2, Corollary 3.2]).

Henceforth, we tacitly assume that every Brelot space considered has a countable base of open sets and that the constant functions are harmonic. (The latter restriction could easily be removed.)

Definition 2 ([CC2, Section 3]). A harmonic morphism $\varphi: X \rightarrow Y$ between Brelot spaces $X$ and $Y$ is said to be of type $\mathrm{Bl}$ if, for any open 
subset $V$ of $Y$ and any locally bounded potential $p$ on $V, p \circ \varphi$ is a potential on $\varphi^{-1}(V)$ (when nonempty).

A harmonic morphism $\varphi: X \rightarrow Y$ is of type $\mathrm{Bl}$ if and only if the natural extension of $\varphi$ to the Wiener compactifications of $X$ and $Y$ maps the harmonic boundary of the former compactification onto the Wiener ideal boundary of the latter (see [Me, Theorem 5.3.3]).

The harmonic morphisms of type $\mathrm{Bl}$ generalize the holomorphic maps of type $\mathrm{Bl}$ between Riemann surfaces introduced by Heins [Hs] (see also [CC1]). When $X$ and $Y$ are the open unit disk $\mathbb{D}$ in $\mathbb{C}$, the holomorphic maps of type $\mathrm{Bl}$ (in the sense of Heins) are the functions representable as a Blaschke product.

In particular, if a harmonic morphism $\varphi: X \rightarrow Y$ between Brelot spaces is a proper map, ${ }^{*}$ then $\varphi$ is of type Bl. Indeed, for any relatively compact open set $V \subset Y, \varphi^{-1}(V)$ is relatively compact and hence of class $\mathfrak{N}$ (see [CC2, Section 3.3], and so [CC2, Theorem 3.10(c)] applies.

In view of [CC2, Theorem 3.4], every bijective harmonic morphism $\varphi$ : $X \rightarrow Y$ is proper (and hence of type $\mathrm{Bl}$ ) because $\varphi^{-1}: Y \rightarrow X$ is a harmonic morphism, and thus, in particular, continuous.

Every harmonic morphism of type $\mathrm{Bl}$ from a connected Brelot space is nonconstant (see [CC2, p. 26]). (Alternatively, take for $V$ in Definition 2 a $\mathfrak{P}$-set on $Y{ }^{\dagger}$ and take for $p$ a locally bounded potential greater than zero on $V$. If $\varphi$ were constant, then $p \circ \varphi$ would be a constant greater than zero on $\varphi^{-1}(V)$, hence harmonic there, and thus not a potential.)

If $\varphi: X \rightarrow Y$ is of type $\mathrm{Bl}$ and if $Y$ is connected, then every closed set contained in $Y \backslash \varphi(X)$ is polar (see [CC2, Corollary 3.7]).

Not every surjective open locally injective harmonic morphism $\varphi: X \rightarrow Y$ is of type $\mathrm{Bl}$, as shown by the following example in which $\varphi$ is an analytic function of a complex variable.

ExAmple 1. With $\mathbb{D}$ denoting the open unit disk in $\mathbb{C}$, let $X=\{x \in$ $\mathbb{D} \backslash\{0\}:|\arg x|<2 \pi / 3\}$, let $Y=X^{2}=\mathbb{D} \backslash\{0\}$, and let $\varphi(x)=x^{2}(x \in X)$. Then $\varphi: X \rightarrow Y$ is a surjective open locally injective harmonic morphism,

${ }^{*}$ A continuous map $\varphi: X \rightarrow Y$ is said to be proper if $\varphi^{-1}(K)$ is compact in $X$ for every compact set $K \subset Y$; here compact may be replaced equivalently by relatively compact (twice).

${ }^{\dagger} \mathrm{A} \mathfrak{P}$-set in a Brelot space $Y$ is an open set on which there exists a potential greater than zero; $Y$ is always covered by $\mathfrak{P}$-sets (see, e.g., [CC3, Theorem 2.3.3]). If the entire space $Y$ is a $\mathfrak{P}$-set, then $Y$ is termed a $\mathfrak{P}$-Brelot space, or a strong Brelot space. 
but not of type Bl. To see this, let $g$ denote the Green function on $\mathbb{D}$ with pole at $-1 / 2$. Since zero is polar in $\mathbb{C}$ and since $g$ is finite and continuous near zero, $g \mid Y$ is also a potential on $Y=\mathbb{D} \backslash\{0\}$, and hence $p:=\min \{1, g \mid Y\}$ is a bounded potential on $Y$. But $p \circ \varphi$ is not a potential on $X$ because it has a continuous extension greater than zero to either of the two boundary segments $\{x \in \mathbb{D} \backslash\{0\}: \arg x= \pm 2 \pi / 3\}$, each of which has nonzero harmonic measure with respect to $X$.

TheOREM 1. Let $\varphi: X \rightarrow Y$ denote a surjective nonconstant harmonic morphism between connected Brelot spaces $X$ and $Y$. For the "if" parts of assertions (a), (b), (c), and (e) below, suppose that the points of $Y$ are polar. For the "only if" part of (b) and the "if" part of (c), suppose in addition that $Y$ satisfies the axiom of polarity, and, for the "if" parts of (d) and (e), suppose that $\varphi$ is an open map of type $\mathrm{Bl} .^{*}$ Finally, suppose for assertion (e) that both $X$ and $Y$ satisfy the axiom of domination.

(a) A set $V \subset Y$ is finely open if and only if the preimage $\varphi^{-1}(V)$ is finely open in $X$. In other words, $\varphi: X \rightarrow Y$ is fine-to-fine open (being surjective) and fine-to-fine continuous.

(b) $A$ set $E \subset Y$ is thin at a point $y \in Y$ if and only if $\varphi^{-1}(E)$ is thin at some (and hence any) point of the fiber $\varphi^{-1}(y) \subset X$.

(c) $A$ set $E \subset Y$ is polar if and only if $\varphi^{-1}(E)$ is polar in $X$.

(d) A function $f$ defined on an open set $V \subset Y$ is superharmonic if and only if $f \circ \varphi$ is superharmonic on $\varphi^{-1}(V)$.

(e) A function $f$ defined on a finely open set $V \subset Y$ is finely superharmonic if and only if $f \circ \varphi$ is finely superharmonic on $\varphi^{-1}(V)$.

Recall that a harmonic space $Y$ is said to satisfy the axiom of polarity if a set $E \subset Y$ is polar provided that $E$ is thin at every point of $Y$ (the converse always holds) or, equivalently, that $E$ is thin at every point of $E$ itself (see [CC3, Theorem 9.1.1]). A harmonic space $X$ is said to satisfy the axiom of domination (axiom D)-respectively, the strong axiom of domination (axiom $\bar{D})$-if, for any locally bounded (resp., finite) potential $p$ on an open set $U \subset X$ and for any hyperharmonic function $u \geq 0$ on $U$ such that $p \leq u$ on the harmonic support ${ }^{\dagger}$ of $p$, we have $p \leq u$ on all of $U$. Equivalently, a locally bounded (resp., finite) potential on $U$ is continuous provided that

\footnotetext{
*Actually, openness of $\varphi$ is not required for (e) in view of Theorem 4(e).

${ }^{\dagger}$ The harmonic support (often called the superharmonic support) of a function $p$ defined on an open set $U$ is the complement of the largest open subset of $U$ on which $p$ is harmonic.
} 
its restriction to its harmonic support is continuous (see [CC3, Theorem 9.2.1], [Jan, Section 2]).* Axiom $D$ implies the axiom of polarity (see [CC3, Corollary 9.2.3]).

In Hansen [Ha], our hypothesis for the "if" part of (d) in Theorem 1, that $\varphi$ be of type $\mathrm{Bl}$, is replaced by a stronger more concrete pair of hypotheses, although extended so as to also apply locally (see [Ha, Korollar 1.7]). The "if" part of (e) in Theorem 1 seems to be new.

Concerning (d) and (e), note furthermore that every nonconstant harmonic morphism $\varphi: X \rightarrow Y$ between connected Brelot spaces $X$ and $Y$ is an open map provided that the points of $Y$ are strongly polar (see [F5, Theorem 4], [F6]).

Definition 3. A point $y$ of a Brelot space $Y$ is called strongly polar if every superharmonic function $s$ on some open neighborhood $V$ of $y$ which is harmonic on $V \backslash\{y\}$, but not at $y$, takes the value $+\infty$ at $y$.

By the Riesz decomposition theorem, it suffices to consider potentials $s$ on $V$. Every strongly polar point $y \in Y$ is polar. To see this, choose an open set $V \subset Y$ on which there exists a potential $p>0$. We may assume that $p(y)<+\infty$ (otherwise, $y$ is already polar). Then $s:={ }^{V} \widehat{R}_{p}^{\{y\}}$ (sweeping relative to $V$ ) is a potential on $V$, and $s$ is harmonic on $V \backslash\{y\}$, hence on all of $V$ because $y$ is strongly polar and yet $s(y) \leq p(y)<+\infty$. Thus, $s \equiv 0$ on $V$. Since $k p \nearrow+\infty$ pointwise on $V$ as $k \nearrow \infty(k \in \mathbb{N})$, we have ${ }^{V} \widehat{R}_{k p}^{\{y\}} \nearrow{ }^{V} \widehat{R}_{+\infty}^{\{y\}}$ pointwise on $V$ by [CC3, Corollary 4.2.1 and Section 5.1]. Note that ${ }^{W} \widehat{R}_{p}^{\{y\} \cap W} \equiv 0$ on the open sets $W=V$ and $W=Y \backslash\{y\}$ (sweeping relative to $W$ ). Since $V \cup W=Y$, it follows that $\{y\}$ is indeed polar (see [CC3, p. 142]).

If $Y$ even satisfies the strong axiom of domination $\bar{D}$, then strongly polar points of $Y$ are the same as polar points (see [F5], [F6]). Suppose that $y \in Y$ is polar, and let $V$ and $s$ be as in Definition 3. If $s(y)<+\infty$, then, by axiom $\bar{D}, s$ would be finite and continuous on $V$ and hence harmonic on all of $V$ by the removable singularity theorem, $\{y\}$ being polar (see, e.g., [CC3, Corollary 6.2.5]). Consequently, $s(y)=+\infty$, and $y$ is indeed strongly polar. Alternatively, use the former definition of axiom $\bar{D}$, taking for $u$ a constant.

${ }^{*}$ The hypothesis in [Jan] that the harmonic spaces in question have the property of nuclearity is fulfilled, in particular, by the present Brelot space (see [CC3, Corollary 11.1.2]). 
The following well-known example already shows that assertion (a) of Theorem 1 may break down if $Y$ contains nonpolar points.

ExAmple 2. Consider a tripod, that is, the union $X$ of three copies $X_{1}, X_{2}, X_{3}$ of the interval [0,1[, glued together at the point 0 (henceforth denoted by $o$ ) of $X$, but otherwise pairwise disjoint. A continuous realvalued function $f$, defined on a connected open subset $V$ of $X$, is said to be harmonic if $f$ is affine-linear on $V$ when $o \notin V$ and if the one-sided derivatives of $f \mid V \cap X_{j}$ at $o(j \in\{1,2,3\})$ have the sum 0 when $o \in V$. It is easily verified that $X$ and hence $Y:=X_{1} \cup X_{2}$ are connected Brelot spaces satisfying axiom $D$ (and even axiom $\bar{D}$ ). The fine topology on $X$ clearly equals the standard topology. Define $\varphi: X \rightarrow Y$ by $\varphi(x)=x$ for $x \in Y$ and by $\varphi(x)=o$ for $x \in X_{3}$. Then $\varphi$ is a surjective nonconstant harmonic morphism, but the points of $Y$ are all nonpolar; and $\varphi$ is neither open nor fine-to-fine open because $X_{3} \backslash\{o\}$ is open and hence finely open on $X$, whereas $\varphi\left(X_{3} \backslash\{o\}\right)=\{o\}$ fails to be even finely open. It is easily verified that (d) and (e) hold true, but $\varphi$ is not of type Bl.

Thus, the requirement for the (equivalent) "if" parts of assertions (d) and (e) of Theorem 1, that $\varphi$ be of type $\mathrm{Bl}$, is not a necessary condition (see also Remark 1 below). This also appears from Example 1 above, where $\varphi$ is surjective open and locally injective, so [CC2, Theorem 3.4] shows that the locally defined inverse $\varphi^{-1}$ is a harmonic morphism, and hence $(f \circ \varphi) \circ \varphi^{-1}=f$ is superharmonic (on $Y$ ) along with $f \circ \varphi$ (on $X$ ) by the "only if" part of (d), valid according to [CC2, Corollary 3.2].

Every Riemannian manifold is a Brelot space satisfying axiom $\bar{D}$, the harmonic functions being understood as the local solutions of class $C^{2}$ to the Laplace-Beltrami equation (see [He, chapitre VII, théorème 36.2]; for the validity of axiom $D$, see also [CC3, Theorem 3.2.1 and Exercise 9.2.9]). (In [CC3], a harmonic space, in particular a Brelot space, is allowed to have a compact underlying space such as the Riemannian manifold $\mathbb{S}^{N-1}$ in Theorem 3.)

REMARK 1. It does not seem likely that the requirements for the "if" parts of assertions (d) and (e) of Theorem 1 (i.e., that $\varphi$ be open and of type $\mathrm{Bl}$ ) can simply be omitted in the stated general setting. It may be noted, however, that in the particular case where $X$ and $Y$ are Riemannian manifolds, every nonconstant harmonic morphism $\varphi: X \rightarrow Y$ is open (see [F6], [BW, p. 112]), and (d) and (e) do hold in the particular case of $C^{2}$ smooth functions $f$ (and hence $f \circ \varphi$ because $\varphi$ is $C^{2}$ here) without any 
extra assumption such as $\varphi$ being of type Bl. Alternatively, this follows from [F4, Lemma 4] because the continuous dilation $\lambda$ is greater than zero on a dense set according to [F4, Lemma 3], the regular (i.e., noncritical) points of a harmonic morphism being precisely the points where $\lambda>0$ (see [F4, Definition 5 and Theorem 7] or [BW, Sections 4.2, 4.3]).

Proof of Theorem 1. (a) The "only if" part of (a) asserts that $\varphi$ is continuous as a map from $X$ into $Y$ with their respective fine topologies. For any open subset $V$ of $Y$, any hyperharmonic function $v$ on $V$, and any real constant $\alpha$, the set

$$
(V, v, \alpha):=\{y \in V: v(y)<\alpha\}
$$

is finely open in $V$ because $v$ is finely continuous. The preimage

$$
\varphi^{-1}((V, v, \alpha))=\left\{x \in \varphi^{-1}(V):(v \circ \varphi)(x)<\alpha\right\}
$$

is finely open in $X$ because $v \circ \varphi$ is hyperharmonic according to [CC2, Theorem 3.1], and hence finely continuous. The claim follows because the sets $(V, v, \alpha)$ form a subbase for the fine topology on $Y$, as $V, v$, and $\alpha$ vary.

According to [CC2, Theorem 3.5], $\varphi$ is an open map from $X$ into $Y$ with their fine topologies because $\varphi$ is a nonconstant harmonic morphism, $X$ is connected, and the points of $Y$ are polar when dealing with the "if" part of (a), which now follows since $\varphi$ is surjective, and hence $V=\varphi\left(\varphi^{-1}(V)\right)$.

(b) For the "only if" part, suppose that $E$ is thin at some point $y \in Y$. If $y \notin E$, then there exists a finely open set $V \subset Y$ such that $y \in V \subset Y \backslash E$. According to the "only if" part of $(\mathrm{a}), \varphi^{-1}(V)$ is finely open in $X$. Clearly, $\varphi^{-1}(V)$ does not meet $\varphi^{-1}(E)$, but does contain every point $x \in \varphi^{-1}(y) \subset$ $X \backslash \varphi^{-1}(E)$, so $\varphi^{-1}(E)$ is indeed thin at $x$. If instead $y \in E$, then the thinness of $E$ at $y$ amounts to $\{y\}$ and $E \backslash\{y\}$ being thin at $y$, and hence $y$ is polar by the axiom of polarity. Therefore, the preimage $\varphi^{-1}(y)$ is likewise polar (see [CC2, Theorem 3.2]), and hence thin at any point $x \in \varphi^{-1}(y)$. As shown above, the set $\varphi^{-1}(E \backslash\{y\})=\varphi^{-1}(E) \backslash \varphi^{-1}(y)$ is likewise thin at $x$, and so is therefore altogether $\varphi^{-1}(E)$, thus establishing the "only if" part of (b).

For the "if" part of (b), let $y \in Y$, and suppose that $\varphi^{-1}(E)$ is thin at some point $x \in \varphi^{-1}(y)$. If $y \notin E$, then $x \notin \varphi^{-1}(E)$, and there exists a finely open set $U \subset X$ such that $x \in U \subset X \backslash \varphi^{-1}(E)$. Because $\varphi$ is fine-to-fine open, by the "if" part of (a) (valid here because the points of $Y$ are supposed to be polar), $\varphi(U)$ is finely open in $Y$ and $y=\varphi(x) \in \varphi(U) \in Y \backslash E$, so $E$ is indeed thin at $y$ in $Y$. If instead $y \in E$, then $\varphi^{-1}(E \backslash\{y\})$ is thin at $y$ along 
with $\varphi^{-1}(E)$, and it follows by the above that $E \backslash\{y\}$ is thin at the polar point $y$. In particular, $\{y\}$ is thin at $y$ (see, e.g., [CC3, Corollary 6.3.2]), and so is altogether $E=(E \backslash\{y\}) \cup\{y\}$.

(c) If $E$ is polar in $Y$, then $\varphi^{-1}(E)$ is polar in $X$, again by [CC2, Theorem 3.2]. Conversely, if $\varphi^{-1}(E)$ is polar in $X$, hence thin at any point of $X$, then $E$ itself is thin at any point of $Y$ by the "if" part of (b). By the axiom of polarity for $Y$, assumed at this point, we infer that $E$ is polar.

(d) The "only if" part holds according to [CC2, Corollary 3.2]. We show that the "if" part holds provided that $\varphi$ is surjective open and of type Bl. Suppose that $V$ is open in $Y$ and that $f \circ \varphi$ is superharmonic on $\varphi^{-1}(V)$ (which is open, by continuity of $\varphi$ ). In particular, $f \circ \varphi$ is lower semicontinuous and greater than $-\infty$ on $\varphi^{-1}(V)$ and is finite on a dense subset $D$ of $\varphi^{-1}(V)$. Since $\varphi$ is surjective and open, it follows that $f$ is lower semicontinuous and greater than $-\infty$ on $V$. And $f$ is finite on $\varphi(D)$, which is dense in $\varphi\left(\varphi^{-1}(V)\right)=V$ because $\varphi$ is surjective and continuous. For the proof that $f$ is hyperharmonic and hence actually superharmonic on $V$, we may assume that $V$ is a $\mathfrak{P}$-set. Thus there exists a potential $q>0$ on $V$, and hence also a bounded potential $p>0$ on $V$ (e.g., $p=\min \{q, 1\}$ ).

Consider a regular set $W$ of compact closure $\bar{W} \subset V$ and a subfunction $v$ for $f$ on $W$. Then $f$ and $-v$ are bounded from below on $W$, and we may therefore assume (after multiplying $p$ by a suitable constant greater than zero) that $f-v \geq-p$ on $W$. It follows that

$$
f \circ \varphi-v \circ \varphi \geq-p \circ \varphi \quad \text { on } \varphi^{-1}(W)
$$

and that $p \circ \varphi$ is a (bounded) potential on $\varphi^{-1}(V)$ because $\varphi$ is supposed to be of type $\mathrm{Bl}$ (see Definition 2). Furthermore, $v \circ \varphi$ is subharmonic on $\varphi^{-1}(W)$ (again by [CC2, Corollary 3.2], $\varphi$ being nonconstant) and we obtain (since $\varphi$ is continuous and $v$ is a subfunction for $f$ on $W$ )

$$
\limsup _{\varphi^{-1}(W) \ni x \rightarrow z}(v \circ \varphi)(x) \leq \limsup _{W \ni y \rightarrow \varphi(z)} v(y) \leq f(\varphi(z))=(f \circ \varphi)(z)
$$

for every $z \in \partial \varphi^{-1}(W)=\overline{\varphi^{-1}(W)} \backslash \varphi^{-1}(W)$, for then $\varphi(z) \in \bar{W} \backslash W=\partial W$. Since $f \circ \varphi$ is lower semicontinuous on $\varphi^{-1}(V) \supset \varphi^{-1}(\bar{W}) \supset \overline{\varphi^{-1}(W)}$, it follows that

$$
\liminf _{\varphi^{-1}(W) \ni x \rightarrow z}(f \circ \varphi-v \circ \varphi)(x) \geq(f \circ \varphi)(z)-(v \circ \varphi)(z) \geq 0
$$


for $z \in \partial \varphi^{-1}(W)$. This, together with (1), implies by [Ba, Korollar 2.4.3] that $f \circ \varphi-v \circ \varphi \geq 0$ on $\varphi^{-1}(W)$, and hence $f \geq v$ on $W$ because $\varphi$ is surjective. By varying the subfunction $v$ for $f$ on $W$, we infer that $f \geq H_{f}^{W}$ on $W$, and so, by varying $W$, that the lower semicontinuous function $f$ on $V$ is hyperharmonic on $V$, and indeed superharmonic there, $f$ being finite on a dense subset of $V$, as shown in the preceding paragraph. Thus the "if" part of (d) indeed holds provided that the harmonic morphism $\varphi: X \rightarrow Y$ is surjective open and of type Bl.

(e) To prove the "only if" part, let $V$ denote a finely open subset of $Y$. If a function $f$ is finely superharmonic on $V$, then $f \circ \varphi$ is finely superharmonic on $\varphi^{-1}(V)$, which is a finely open subset of $X$ by the "only if" part of (a). This follows from [F3, Corollary 6] which is applicable because our nonconstant harmonic morphism $\varphi: X \rightarrow Y$ is also a finely harmonic morphism (mapping) by [F3, Definition 2 and Section 3(e)] (see also Section 4 below), and because the connected space $X$ is also a finely connected finely open subset of $X$ since $X$ satisfies axiom $D$ (see [F1], [F2, p. 88]).

Having thus shown that the "only if" parts of (d) and (e) always hold, we now show that the "if" parts of (d) and (e) are equivalent statements (when the points of $Y$ are polar, the harmonic morphism $\varphi$ is open, and $X$ and $Y$ satisfy axiom $D$, but without assuming that $\varphi$ be of type $\mathrm{Bl}$ ). First, the "if" part of (e), whenever valid, implies that of (d). Indeed, the given open set $V \subset Y$ is also finely open. A superharmonic function of the form $f \circ \varphi$ on the open set $\varphi^{-1}(V)$ is also finely superharmonic on that set (which is also finely open) by [F2, Theorem 8.7 and Section 10.4], the components of $\varphi^{-1}(V)$ being the same as the fine components (see [F1], [F2, p. 88]). From the "if" part of (e) it follows that $f$ is finely superharmonic on the open set $V$, and therefore superharmonic there by [F2, Theorem 9.8], $f$ being locally bounded from below on $V$ along with the superharmonic function $f \circ \varphi$ on $\varphi^{-1}(V)$ because $\varphi$ is a surjective open map.

Conversely, the "if" part of (d), whenever valid, implies the "if" part of (e) (this implication does not require that $\varphi$ be open). We may assume that $Y$ is a $\mathfrak{P}$-space. Consider a finely open set $V \subset Y$ and a function $f$ on $V$ such that $f \circ \varphi$ is finely superharmonic on $\varphi^{-1}(V)$, which is finely open in $X$ by the "if" part of (a) (valid here since the points of $Y$ are supposed to be polar). For the proof that $f$ itself then is finely superharmonic on $V$, suppose to begin with that $f$ and hence $f \circ \varphi$ are bounded from above. Recall that a relatively compact finely open subset $W$ of $V$ is said to be regular if $Y \backslash W$ is a base, that is, $Y \backslash W$ is non-thin at each of its points, 
or, equivalently, $\varepsilon_{y}^{Y \backslash W}=\varepsilon_{y}$ for every $y \in Y \backslash W$ (see, e.g., [CC3, Theorem 7.1.1] or [F2, Section 3.3]). Here $\varepsilon_{y}^{Y \backslash W}$ denotes the swept-out of the Dirac measure $\varepsilon_{y}$ on $Y \backslash W$. This harmonic measure $\varepsilon_{y}^{Y \backslash W}$ for $W$ is carried by $Y \backslash W$ (see, e.g., [CC3, Theorem 7.2.1]) (noting that a regular finely open set $W$ is an $F_{\sigma}$ set as in [CC3, Corollary 7.2.1], and hence measurable).

Being finely superharmonic on $\varphi^{-1}(V), f \circ \varphi$ is finely continuous, bounded from above, and $>-\infty$ on $\varphi^{-1}(V)$ by [F2, Theorem 9.10]. Hence $f$ is finely continuous in view of (a), bounded from above, and greater than $-\infty$ on $V$. (Note that (a) holds here because the points of $Y$ are supposed to be polar.) Consider now a regular finely open set $W$ with closure $\bar{W} \subset V$ such that $f$ is bounded also from below on $\bar{W}$. According to [F2, Theorem 3.10], there exists a bounded finely continuous (auxiliary) function $g$ on $Y$ such that $g=f$ on the fine closure $\bar{W}^{\mathrm{f}}=W \cup b(W)=b(W)$ of $W$ (see, e.g., [F2, Section 3.6]).* Define a function $u: Y \rightarrow \mathbb{R}$ by

$$
u(y)=\int g d \varepsilon_{y}^{Y \backslash W}, \quad y \in Y .
$$

With the (nonstandard) notation of [F2, Definition 4.10], this means that $u=g^{Y \backslash V}$. According to [F2, Lemma 9.3 and Section 8.4], $u$ is bounded and finely continuous on $Y$ and finely harmonic on $W$. Furthermore,

$$
u(y)= \begin{cases}\int f d \varepsilon_{y}^{Y \backslash W}, & y \in W, \\ f(y), & y \in \partial_{\mathrm{f}} W,\end{cases}
$$

the former because $f=g$ on the fine boundary $\partial_{\mathrm{f}} W$ which carries the harmonic measure $\varepsilon_{y}^{Y \backslash W}$ when $y \in W$ (see [F2, Sections 4.7, 4.8]), and the latter because $\varepsilon_{y}^{Y \backslash W}=\varepsilon_{y}$ for $y \in Y \backslash W$, in particular, for $y \in \partial_{\mathrm{f}} W$, where thus $u(y)=g(y)=f(y)$.

By the "only if" part of (e) (settled above), applied to the finely harmonic functions $u$ and $-u$ on $W$, it follows that $u \circ \varphi$ is both finely superharmonic and finely subharmonic on $\varphi^{-1}(W)$, and hence finely harmonic there by [F2, Corollary 1, p. 84]. Moreover, $u \circ \varphi$ is bounded on $\varphi^{-1}(\bar{W})$, along with $u$ on $Y$. Write $s=f-u, s^{-}=\min \{s, 0\}$ on $W$, and denote by $s_{0}^{-}(\leq 0)$ the bounded and (in view of (2)) finely continuous extension of $s^{-}$to $Y$ by zero on $Y \backslash W$. Now apply [F2, Lemma 10.1] with $u, U, v$, and $V$ replaced

\footnotetext{
${ }^{*}$ Rather than introducing this function $g$ and applying [F2, Lemma 9.3], one might alternatively use [F2, Theorem 14.1] on the fine Dirichlet problem.
} 
by $0, X, s \circ \varphi$, and $\varphi^{-1}(W)$, respectively. It follows that $s_{0}^{-} \circ \varphi$ is finely superharmonic on $X$, and hence superharmonic there by [F2, Theorem 9.8]. From the "if" part of $(\mathrm{d})$ we infer that $s_{0}^{-}$is superharmonic on $Y$, and hence finely superharmonic there by [F2, Theorem 8.7], in particular, on $W$, where $s_{0}^{-}=s^{-}$. Because $s_{0}^{-}=0$ on $\partial_{\mathrm{f}} W \subset Y \backslash W$, we have $s_{0}^{-}=s^{-} \geq 0$ on $W$ by the fine boundary minimum principle (see [F2, Theorem 9.1 and Section 8.4]). It follows that $s^{-}=0$, that is, $s \geq 0$, and, consequently, that $f \geq u$ on $W$. In view of (2) this shows, by varying $W$, that indeed $f$ is finely superharmonic on $V$ by [F2, Definition 8.1 and Section 8.4].

Finally, we drop the temporary hypothesis that $f$ be bounded from above. For $n \in \mathbb{N}$, write $f_{n}=\min \{f, n\}$. Then $f_{n} \circ \varphi=\min \{f \circ \varphi, n\}$ is bounded from above and finely superharmonic on $\varphi^{-1}(V)$, the constant $n$ being harmonic on $X$, and hence finely harmonic on $\varphi^{-1}(V)$ by $[\mathrm{F} 2$, Theorem 8.7]. We have therefore shown above that $f_{n}$ itself is finely superharmonic on $V$, and so $f$ is finely hyperharmonic on $V$, being the pointwise limit of the increasing sequence $\left(f_{n}\right)$ of finely hyperharmonic functions (see [F2, Corollary 2, p. 84]). Writing $D=\{y \in V: f(y)<+\infty\}$, we have $\varphi^{-1}(D)=\left\{x \in \varphi^{-1}(V):(f \circ \varphi)(x)<+\infty\right\}$. In view of [F2, Section 10.4], the latter set $\varphi^{-1}(D)$ is finely dense in $\varphi^{-1}(V)$ because $f \circ \varphi$ is finely superharmonic there; and hence $D=\varphi\left(\varphi^{-1}(D)\right)$ is finely dense in $V, \varphi: X \rightarrow Y$ being surjective and moreover fine-to-fine open by the "if" part of (a) (valid here because the points of $Y$ are supposed to be polar). Consequently, $f$ is indeed finely superharmonic on $V$. This completes the proof of Theorem 1 , including the stated equivalence of the "if" parts of (d) and (e).

\section{$\S 2$. Two examples in classical potential theory}

TheOREM 2. The projection $\varphi: \mathbb{R}^{N} \rightarrow \mathbb{R}^{n}(N>n \geq 1)$, given by $\varphi\left(x_{1}, \ldots\right.$, $\left.x_{N}\right)=\left(x_{1}, \ldots, x_{n}\right)$, is a surjective open harmonic morphism of type $B l$, and assertions (a)-(e) of Theorem 1 apply to $X=\mathbb{R}^{N}, Y=\mathbb{R}^{n}$, and to this projection map $\varphi$.

Note that $f \circ \varphi$ is the extension of $f$ from $\mathbb{R}^{n}$ to $\mathbb{R}^{N}$ depending only on $x_{1}, \ldots, x_{n}$. The case $n=N-1$ of assertions (a)-(d) of Theorem 2 is known (see [DL] for (c) and (d), [Ha] for (a)-(d), and [G2] for (a)-(c)). Actually, these quoted results together imply our assertions (a)-(d) in Theorem 2 by repeated application to $N, N-1, \ldots, n+1$ in place of $N$ (or by copying the proof, as noted in [DL, p. 94]). 
Proof of Theorem 2. The spaces $X=\mathbb{R}^{N}$ and $Y=\mathbb{R}^{n}$ are connected Brelot spaces satisfying the axiom of domination $D$ (even the strong one, $\bar{D})$ and, in particular, the axiom of polarity. The projection $\varphi: \mathbb{R}^{N} \rightarrow \mathbb{R}^{n}$ clearly is a surjective and open (hence nonconstant) harmonic morphism.

In the case $N=2, n=1$, where the points of $Y=\mathbb{R}^{1}$ are nonpolar, (a)(c) nevertheless hold, as shown in [G1, Section 2]. Alternatively, note that the fine topology on $Y=\mathbb{R}^{1}$ equals the standard topology, and hence $\varphi$ is fine-to-fine continuous (being of course continuous). To see that $\varphi$ is fine-tofine open, recall that the fine topology is locally connected, (see [F1], [F2, Corollary 9.11]). If $U$ is a fine domain in $X=\mathbb{R}^{2}$ and if $x \in U$, then $\varphi(U)$ is finely connected (along with $U$ ), that is, connected, and so $\varphi(U)$ is an interval containing $\varphi(x)$. If $\varphi(x)$ were an endpoint of $\varphi(U)$, then $U$ would be contained in a half-plane in $\mathbb{R}^{2}$ and bounded by a line through $x$, which is impossible since $U$ is finely open.

With assertion (a) thus established for $N=2, n=1$, the "if" parts of (b) and (c) follow as shown in the proof of Theorem 1, noting that a fiber $\varphi^{-1}(y)$ in $\mathbb{R}^{2}$ is a straight line and hence not thin at points of itself, let alone polar.

For any $N>n \geq 1$, denote by $\varphi_{N, n}$ the projection $\mathbb{R}^{N} \rightarrow \mathbb{R}^{n}$. As just shown, (a)-(c) hold for $\varphi_{2,1}$. When $N \geq 3$, they hold for $\varphi_{N, 2}$ by Theorem 1 , and consequently they also hold for $\varphi_{N, 1}=\varphi_{2,1} \circ \varphi_{N, 2}$.

The "only if" parts of (d) and (e) hold for $N>n \geq 1$ according to Theorem 1. We proceed to verify directly the "if" part of (d). From that will follow the "if" part of (e) as shown in the proof of Theorem 1, noting that the polarity of points of $Y$ missing here is only being used through the valid assertion (a). Afterward, we apply (d) to show that $\varphi$ is of type Bl.

Consider a function $f$ on an open set $V \subset Y$ such that $f \circ \varphi$ is superharmonic on the open set $\varphi^{-1}(V)$. In particular, $f \circ \varphi$ is lower semicontinuous and greater than $-\infty$ on $\varphi^{-1}(V)$, and so therefore is $f$ itself on $V$ because $\varphi$ is surjective and open. For the direct proof that $f$ itself must be superharmonic on $V$ there is the complication that $\varphi: \mathbb{R}^{N} \rightarrow \mathbb{R}^{n}$ is not proper. We therefore begin by replacing $\mathbb{R}^{N-n}$ by the compact $(N-n)$-torus $\mathbb{T}^{N-n}=(\mathbb{R} / \mathbb{Z})^{N-n}$ and hence $X \cong \mathbb{R}^{n} \times \mathbb{R}^{N-n}$ by $X_{*}:=\mathbb{R}^{n} \times \mathbb{T}^{N-n}$, and by replacing the projection $\varphi: X \rightarrow \mathbb{R}^{n}$ by the projection $\varphi_{*}: X_{*} \rightarrow \mathbb{R}^{n}$. The canonical map $\tau: \mathbb{R}^{N-n} \rightarrow \mathbb{T}^{N-n}$ is a local isometry, and so therefore is id $\times \tau: \mathbb{R}^{n} \times \mathbb{R}^{N-n} \rightarrow \mathbb{R}^{n} \times \mathbb{T}^{N-n}$, where id denotes the identity map of $\mathbb{R}^{n}$. In particular, id $\times \tau: X \rightarrow X_{*}$ is a locally injective harmonic morphism between connected Riemannian manifolds (in particular, connected Brelot 
spaces). Because $f \circ \varphi$ is supposed to be superharmonic on the open subset $\varphi^{-1}(V) \cong V \times \mathbb{R}^{N-n}$ of $X \cong \mathbb{R}^{n} \times \mathbb{R}^{N-n}$ and because

$$
f \circ \varphi=\left(f \circ \varphi_{*}\right) \circ(\mathrm{id} \times \tau)
$$

it follows from [CC2, Theorem 3.4], applied locally, that $f \circ \varphi_{*}$ is superharmonic on the open subset $\varphi_{*}^{-1}(V) \cong V \times \mathbb{T}^{N-n}$ of $X_{*}=\mathbb{R}^{n} \times \mathbb{T}^{N-n}$. But $\varphi_{*}$ is a proper map and hence of type $\mathrm{Bl}$ because $\mathbb{T}^{N-n}$ is compact, and it therefore follows from the "if" part of Theorem $1(\mathrm{~d})$, applied with $\varphi$ replaced by $\varphi_{*}$, that $f$ is indeed superharmonic on $V$.

An alternative to this proof of the "if" part of Theorem 2(d) can be readily obtained by adapting the proof of the "if" part of Theorem 3(d) given below.

With the application of the "if" part of (d) of Theorem 2 thus established, we proceed to prove that $\varphi$ is of type Bl. For a given locally bounded potential $p$ on an open set $V \subset Y=\mathbb{R}^{n}$ we show that $p \circ \varphi$ is a potential on the open set $\varphi^{-1}(V) \subset \mathbb{R}^{N}$. By the "only if" part of (d) (see above), we know that $p \circ \varphi$ is superharmonic on $\varphi^{-1}(V)$. Let $h$ be the greatest harmonic minorant of $p \circ \varphi$ on $\varphi^{-1}(V)$. Identifying $\mathbb{R}^{N}$ with $\mathbb{R}^{n} \times \mathbb{R}^{N-n}$, write $x=(y, z)$ with

$$
y=\varphi(x)=\left(x_{1}, \ldots, x_{n}\right) \in \mathbb{R}^{n}, \quad z=\left(x_{n+1}, \ldots, x_{N}\right) \in \mathbb{R}^{N-n} .
$$

For $t \in \mathbb{R}^{N-n}$, consider the harmonic function $h_{t}$ on $\varphi^{-1}(V)$ given by

$$
h_{t}(x)=h_{t}(y, z)=h(y, z+t) \leq p(y)=(p \circ \varphi)(x) .
$$

Clearly, $h_{t} \leq h$, and hence $h_{t}=h$ for every $t \in \mathbb{R}^{N-n}$ (replace $z$ by $z-t$ ). Thus $h(x)=h(y, z)$ is independent of $z \in \mathbb{R}^{N-n}$, so we may write $h(x)=$ $g(y)$ for $x=(y, z) \in \varphi^{-1}(V)$, whereby the function $g$ is defined on $V$, and $h=g \circ \varphi$. By the "if" part of (d), $g$ is harmonic on $V$. Because $g \leq p$, it follows that $g \leq 0$. Thus $h \leq 0$ (in fact, $h=0$ since $h \geq 0$ ), proving that $p \circ \varphi$ is a potential on $\varphi^{-1}(V)$. Thus $\varphi$ is indeed of type $\mathrm{Bl}$.

THEOREM 3. The radial projection $\varphi: \mathbb{R}^{N} \backslash\{0\} \rightarrow \mathbb{S}^{N-1}$ (the unit sphere in $\mathbb{R}^{N}, N>1$ ), given by $\varphi(x)=x /|x|$, is a surjective open harmonic morphism of type Bl, and assertions (a)-(e) of Theorem 1 apply to $X=\mathbb{R}^{N} \backslash$ $\{0\}, Y=\mathbb{S}^{N-1}$, and to the radial projection $\varphi$.

Assertions (b), (c), and at least the "if" part of (d) of Theorem 3 are known (see [DL], [G1]). Further properties of the radial projection are found in $[\mathrm{BW}, \mathrm{p} .102]$. 
Proof of Theorem 3. The spaces $X=\mathbb{R}^{N} \backslash\{0\}$ and $Y=\mathbb{S}^{N-1}$ are connected Brelot spaces satisfying axiom $D$ (and even $\bar{D}$ ), and hence the axiom of polarity. The radial projection $\varphi: \mathbb{R}^{N} \backslash\{0\} \rightarrow \mathbb{S}^{N-1}$ is a surjective open map. Furthermore, $\varphi$ is a harmonic morphism, as seen from the expression for the Laplacian $\Delta$ on $\mathbb{R}^{N} \backslash\{0\}$ in polar coordinates $\left.r=|x| \in\right] 0,+\infty[$ and $\theta=x /|x| \in \mathbb{S}^{N-1}$ :

$$
\Delta u=r^{1-N} \frac{\partial}{\partial r}\left(r^{N-1} \frac{\partial u}{\partial r}\right)+\frac{1}{r^{2}} \Delta^{\mathbb{S}^{N-1}} u
$$

for $u \in C^{2}\left(\mathbb{R}^{N} \backslash\{0\}\right)$, with $\Delta^{\mathbb{S}^{N-1}}$ denoting the Laplace operator on $\mathbb{S}^{N-1}$. In particular,

$$
\Delta(h \circ \varphi)=r^{-2}\left[\left(\Delta^{\mathbb{S}^{N-1}} h\right) \circ \varphi\right]
$$

on $\mathbb{R}^{N} \backslash\{0\}$ for $h \in C^{2}\left(\mathbb{S}^{N-1}\right)$ because $h \circ \varphi$ is a function solely of $\theta \in \mathbb{S}^{N-1}$.

Assertions (a)-(c) are established in the same way as in the proof of Theorem 2, beginning with (a), first for $N=2$ (where the points of $Y=$ $\mathbb{S}^{1}$ are nonpolar and where the fine topology on $Y$ equals the Euclidean topology).

The "only if" parts of (d) and (e) of Theorem 3 hold by the proof of Theorem 1. Unlike the proof of the "if" part of (d) in Theorem 2, it does not seem possible to reduce the "if" part of (d) in Theorem 3 to the case of proper maps, so we proceed differently.

The given superharmonic function $f \circ \varphi$ on the open set $\varphi^{-1}(V) \subset \mathbb{R}^{N} \backslash$ $\{0\}$ (with $V$ open in $\mathbb{S}^{N-1}$ ) is denoted by $s$; then $s$ is also superharmonic in the distributional sense (see, e.g., [AG, Section 4.3]). In terms of the polar coordinates $r=|x|>0$ and $\theta=x /|x| \in \mathbb{S}^{N-1}$, we may write $s=s(r, \theta)=$ $s(\theta), \theta \in V$. We consider test functions $\not \equiv 0$ of the form $(r, \theta) \mapsto u(r) v(\theta)$ with nonnegative functions $u \in C_{0}^{\infty}(] 0,+\infty[)$ and $v \in C_{0}^{\infty}(V)$. In terms of the Riemannian volume measure $d \theta$ on $\mathbb{S}^{N-1}$, the Lebesgue measure on $\mathbb{R}^{N}$ equals $r^{N-1} d r d \theta$, and we obtain in view of (3)

$$
\begin{aligned}
0 \geq & \iint s \Delta(u \otimes v) r^{N-1} d r d \theta \\
= & \int_{0}^{\infty} \frac{d}{d r}\left(r^{N-1} \frac{d u}{d r}\right) d r \int_{V} s(\theta) v(\theta) d \theta \\
& +\int_{0}^{\infty} r^{N-3} u(r) d r \int_{V} s(\theta) \Delta^{\mathbb{S}^{N-1}} v(\theta) d \theta
\end{aligned}
$$


The former integral over $0<r<+\infty$ equals zero because $u$ and hence $r^{N-1} d u / d r$ have compact support. Inserting this and dividing by $\int_{0}^{\infty} r^{N-3} u(r) d r>0$ leads to

$$
\int_{V} s(\theta) \Delta^{\mathbb{S}^{N-1}} v(\theta) d \theta \leq 0
$$

for every nonnegative $v \in C_{0}^{\infty}(V)$, and so $\Delta^{\mathbb{S}^{N-1}} s \leq 0$ in the distributional sense, $\Delta^{\mathbb{S}^{N-1}}$ being formally self-adjoint. It is well known that this inequality amounts to $s=s(\theta)$ being equal $d \theta$-almost everywhere to a superharmonic function $s^{*}$ on $V$. According to the "if" part of (a), which is valid here, the function $s=s(\theta)$ is finely continuous on $V$ because $s=s(r, \theta)=(f \circ \varphi)(x)$ is superharmonic and hence finely continuous for $x \in \mathbb{R}^{N} \backslash\{0\}$. The set $\{\theta \in$ $\left.V: s(\theta) \neq s^{*}(\theta)\right\}$ is therefore a finely open null set on $V$, and hence void.* It follows that $f(y)=s(\theta)=s^{*}(\theta)$ is indeed superharmonic on $V \subset \mathbb{S}^{N-1}$.

Having thus established the "if" part of $(\mathrm{d})$, we deduce the "if" part of (e) as in the proof of Theorem 1 (or Theorem 2), noting again that the missing polarity of points of $Y$ is only used through the valid assertion (a). Finally, we apply (d) to prove that $\varphi: \mathbb{R}^{N} \backslash\{0\} \rightarrow \mathbb{S}^{N-1}$ is of type Bl. Given a locally bounded potential $p$ on an open set $V \subset Y=\mathbb{S}^{N-1}$, we prove that $p \circ \varphi$ is a potential on $\varphi^{-1}(V)$ in a way similar to that described toward the end of the proof of Theorem 2. Let $h$ be the greatest harmonic minorant of $p \circ \varphi$ on $\varphi^{-1}(V)$. Replace the previous $t \in \mathbb{R}^{N-n}$ and $h_{t}$ by $0<t<+\infty$ and

$$
h_{t}(x)=h(t x) \leq(p \circ \varphi)(x)
$$

for $x \in \varphi^{-1}(V)$, and deduce that $h_{t}=h$ only depends on $\varphi(x)=x /|x|=\theta \in$ $V \subset \mathbb{S}^{N-1}$, and so on.

\section{$\S 3$. Finely harmonic morphisms}

We close with a version of Theorem 1 in which the Brelot space $X$ is replaced by a fine domain (i.e., a finely connected finely open set) $X$ in an ambient Brelot space $\Omega$ satisfying axiom $D$. The target $Y$ is likewise supposed to be a Brelot space satisfying axiom $D$. Both $\Omega$ and $Y$ are supposed

${ }^{*}$ It is indeed well known that every finely open Lebesgue null set $U \subset \mathbb{R}^{N}$ is void. For the proof, we may assume that there exists a superharmonic function $u$ on an open set $\Omega \subset \mathbb{R}^{N}$ such that $U=\{x \in \Omega: u(x)<0\}$. Then $\min \{u, 0\}$ is superharmonic on $\Omega$ and equals zero almost everywhere on $\Omega$, hence actually everywhere on $\Omega$ (see, e.g., [AG, Corollary 3.2.7]). Thus $u \geq 0$ on $\Omega$, and so $U$ is indeed void. 
to have countable bases of open sets, and the constant functions on $\Omega$ and on $Y$ are supposed to be harmonic.

Definition 4. A fine-to-fine continuous map $\varphi: X \rightarrow Y$ is called a finely harmonic morphism if, for any finely harmonic function $f$ on a finely open set $V \subset Y, f \circ \varphi$ is finely harmonic on $\varphi^{-1}(V)$ (when nonempty).

We show that one may equivalently replace finely harmonic function by finely hyperharmonic function in the case of $f$ and $f \circ \varphi$ in Definition 4. Clearly, this finely hyperharmonic version of Definition 4, when applied to $\pm f$, implies Definition 4 as it stands (the finely harmonic version). Next, Definition 4 trivially implies the mixed topology version thereof proposed in [F3, Definition 2], where the fine topology on $Y$ is replaced by the initially given topology and where $f$ is supposed to be harmonic on an (initially) open set $V \subset Y$ (see [F2, Theorem 8.7]). And finally, this mixed topology version of Definition 4 implies the finely hyperharmonic version according to the "only if" part of [F3, Theorem 5]. These three versions of Definition 4 are thus equivalent.

In the particular case where $X$ is a connected open subset of $\Omega$, and hence itself a connected Brelot space (as in Section 1 above), then every harmonic morphism $\varphi: X \rightarrow Y$ (see Definition 1) is also a finely harmonic morphism (see Definition 4). This is shown by using the mixed topology version of Definition 4 and applying [F2, Theorem 8.7] again. If the points of $Y$ are polar, then harmonic and finely harmonic morphisms (from the connected open set $X \subset \Omega$ to $Y$ ) are the same, as shown by Laine [La1, Theorem 2.1.5].

The concept of type $\mathrm{Bl}$ has an analogue for finely harmonic morphisms. Recall that a fine potential on a finely open subset of a Brelot space satisfying axiom $D$ is defined as a finely superharmonic function greater than or equal to zero for which every finely subharmonic minorant is less than or equal to zero (see [F2, Definition 10.5]). For a finely superharmonic function greater than or equal to zero on a finely open subset of $\mathbb{R}^{N}$ with $N \geq 3$ to be a fine potential, it is not sufficient to require that every finely harmonic minorant be less than or equal to zero (see $[\mathrm{GH}]$ ).

Definition 5. A finely harmonic morphism $\varphi: X \rightarrow Y$ ( $X$ finely open in $\Omega$ ) is said to be of fine type $\mathrm{Bl}$ if, for any finite fine potential $p$ on a finely open set $V \subset Y, p \circ \varphi$ is a (finite) fine potential on $\varphi^{-1}(V)$ (which is finely open, and assumed nonempty). 
In the case where $X$ is an open subset of $\Omega$, a harmonic morphism $\varphi: X \rightarrow$ $Y$ of fine type $\mathrm{Bl}$ (see Definition 5) is also of type $\mathrm{Bl}$ (see Definition 2) in view of [F2, Corollary 10.12]; the converse seems to remain open (even if the points of $Y$ are polar).

We are now prepared to essentially extend Theorem 1 (except, of course, for part (d) involving only the initial topology) to the present case of a finely harmonic morphism.

THEOREM 4. Let $\varphi: X \rightarrow Y$ denote a surjective nonconstant finely harmonic morphism from a fine domain $X$ in a Brelot space $\Omega$ to a Brelot space $Y$, and suppose that $\Omega$ and $Y$ both satisfy axiom D. For the "if" parts of assertions (a), (b), (c), and (e) of Theorem 1, suppose that the points of $Y$ are polar. For the "if" part of (e), suppose that $\varphi$ is of fine type Bl. Then $Y$ is connected, and assertions (a)-(c) and (e) of Theorem 1 hold in the present setting.

Proof. Recall that the axiom of polarity holds here, being a consequence of axiom $D$ (see [CC3, Corollary 9.2.3]).

(a) According to [F3, Theorems 5 and 7], $\varphi$ is indeed fine-to-fine continuous and fine-to-fine open. This implies the "only if" part of (a) and also the "if" part, $\varphi$ being surjective. It follows moreover that $\varphi(X)=Y$ is finely connected (along with $X$ ) and, in particular, connected.

(b) Here, (b) is derived from (a) in the same way as in the proof of Theorem 1(b).

(c) If $E$ is polar in $Y$, then $\varphi^{-1}(E) \subset X \subset \Omega$ is polar in $\Omega$ by [F3, Theorem 6]. The converse is established in the same way as in the proof of Theorem 1(c).

(e) The "only if" part holds according to [F3, Corollary 6]. For the proof of the "if" part of (e), we proceed much as in the proof of the "if" part of Theorem $1(\mathrm{~d})$, now in the fine setting. We may assume that $Y$ is a $\mathfrak{P}$-space. Suppose that $V$ is finely open in $Y$ and that $f \circ \varphi$ is finely superharmonic on $\varphi^{-1}(V)$ (finely open, by fine-to-fine continuity of $\varphi$ ). In particular, $f \circ \varphi$ is finely lower semicontinuous and $>-\infty$ on $\varphi^{-1}(V)$, and finite on a finely dense subset $D$ of $\varphi^{-1}(V)$. Since $\varphi$ is surjective and fine-to-fine open, it follows that $f$ is finely lower semicontinuous and $>-\infty$ on $V$. And $f$ is finite on $\varphi(D)$, which is finely dense in $\varphi\left(\varphi^{-1}(V)\right)=V$ because $\varphi$ is surjective and fine-to-fine continuous. For the proof that $f$ is finely hyperharmonic (and hence actually finely superharmonic) on $V$, we may choose a fine potential 
$q>0$ on $V$, hence also a bounded fine potential $p>0$ on $V$, (e.g., $p=$ $\min \{q, 1\})$.

For a given point $y \in V$, consider a finely open set $W \ni y$ of compact closure $\bar{W} \subset V$ such that $p>(1 / 2) p(y)$ on $W$, and consider a fine subfunction $v$ for $f$ on $W$ such that $v$ is bounded from above on $W$ (see [F2, Section 14.3]). After multiplying $p$ by a suitable constant $>0$, we may assume that $f-v \geq-p$ on $W$. It follows that (1) holds and that $p \circ \varphi$ is a (bounded) fine potential on $\varphi^{-1}(W)$ because $\varphi$ is supposed to be of fine type $\mathrm{Bl}$ (see Definition 5). Furthermore, $v \circ \varphi$ is finely subharmonic on $\varphi^{-1}(W)$ by the "only if" part of (e), and we obtain (since $\varphi$ is fine-to-fine continuous and $v$ is a fine subfunction for $f$ on $W$ )

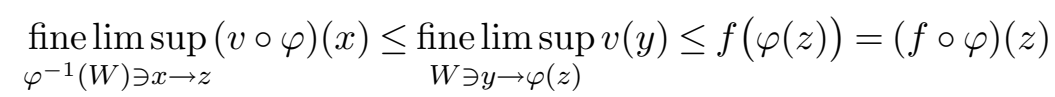

for every $z \in \partial_{\mathrm{f}} \varphi^{-1}(W)={\overline{\varphi^{-1}(W)}}^{\mathrm{f}} \backslash \varphi^{-1}(W)$. (We denote by $\partial_{\mathrm{f}} A$ the fine boundary and by $\bar{A}^{\mathrm{f}}$ the fine closure of a subset $A$ of $X$.) Since $f \circ \varphi$ is finely lower semicontinuous on $\varphi^{-1}(V) \supset \varphi^{-1}\left(\bar{W}^{\mathrm{f}}\right) \supset \overline{\varphi^{-1}(W)} \mathrm{f}$, it follows that

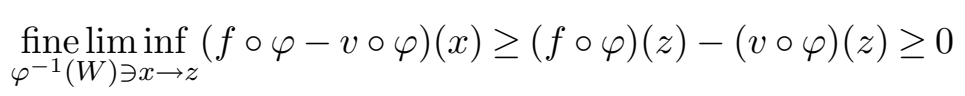

for $z \in \partial_{\mathrm{f}} \varphi^{-1}(W)$. Together with (1), this implies, by [F2, Theorem 10.8] (replacing here [Ba, Korollar 2.4.3]), that $f \circ \varphi-v \circ \varphi \geq 0$ on $\varphi^{-1}(W)$, and hence $f \geq v$ on $W$ because $\varphi$ is surjective. By varying the fine subfunction $v$ for $f$ on $W$, we infer that $f \geq \underline{H}_{f}^{W}$ on $W$ (see [F2, Section 14.3] concerning $\underline{H}_{f}$ ), and hence

$$
f(y) \geq \underline{H}_{f}^{W}(y)=\int_{*} f d \varepsilon_{y}^{\Omega \backslash W}
$$

by [F2, Theorem 14.6]. The finely upper semicontinuous function $-f$ differs only on a polar set from its base, which is a Borel measurable function (see [Do]), so the above lower integral can be replaced by the actual integral. By varying $y$ and $W$, it follows that the finely lower semicontinuous function $f$ on $V$ is finely hyperharmonic on $V$, and indeed finely superharmonic there by [F2, Section 10.4], $f$ being finite on a finely dense subset of $V$, as shown in the preceding paragraph. Thus the "if" part of (e) indeed holds provided that the finely harmonic morphism $\varphi: X \rightarrow Y$ is surjective, nonconstant, and of fine type $\mathrm{Bl}$. 
Acknowledgment. The author wishes to thank Professor Wolfhard Hansen for a careful reading of the manuscript and, in particular, for providing a simpler proof of the Bl property at the end of Theorems 2 and 3.

\section{REFERENCES}

[AG] D. H. Armitage and S. J. Gardiner, Classical Potential Theory, Springer, Berlin, 2001.

[BW] P. Baird and J. C. Wood, Harmonic Morphisms Between Riemannian Manifolds, Clarendon Press, Oxford, 2003.

[Ba] H. Bauer, Harmonische Räume und ihre Potentialtheorie, Lecture Notes in Math. 22, Springer, Berlin, 1966.

[BH] J. Bliedtner and W. Hansen, Potential Theory - An Analytic and Probabilistic Approach to Balayage, Springer, Berlin, 1986.

[Br] M. Brelot, Lectures on Potential Theory, Tata Inst. Fund. Res., Mumbai, 1960.

[CC1] C. Constantinescu and A. Cornea, Ideale Ränder Riemannscher Flächen, Ergeb. Math. Grenzgeb. 32, Springer, Berlin, 1963.

[CC2] - Compactifications of harmonic spaces, Nagoya Math. J. 25 (1965), 1-57.

[CC3] , Potential Theory on Harmonic Spaces, Grundlehren Math. Wiss., Band 158, Springer, Berlin, 1972.

[DL] J. Deny and P. Lelong, Étude des fonctions sousharmoniques dans un cylindre ou dans un cône, Bull. Soc. Math. France 75 (1947), 89-112.

[Do] J. L. Doob, Applications to analysis of a topological definition of smallness of a set, Bull. Amer. Math. Soc. (N.S.) 72 (1966), 579-600.

[F1] B. Fuglede, Connexion en topologie fine et balayage des mesures, Ann. Inst. Fourier (Grenoble) 21 (1971), 227-244.

[F2] - Finely Harmonic Functions, Lecture Notes in Math. 289, Springer, Berlin, 1972.

[F3] , Finely harmonic mappings and finely holomorphic functions, Ann. Acad. Sci. Fenn. Math. 2 (1976), 113-127.

[F4] , Harmonic morphisms between Riemannian manifolds, Ann. Inst. Fourier (Grenoble) 28 (1978), 107-144.

[F5] , "Harmonic morphisms" in Complex Analysis (Joensuu, 1978), Lecture Notes in Math. 747, Springer, Berlin, 1979, 123-131.

[F6] - Harnack sets and openness of harmonic morphisms, Math. Ann. 241 (1979), 181-186.

[G1] S. J. Gardiner, The Lusin-Primalov theorem for subharmonic functions, Proc. Amer. Math. Soc. 124 (1996), 3721-3727.

[G2] - Growth properties of superharmonic functions along rays, Proc. Amer. Math. Soc. 128 (2000), 1963-1970.

[GH] S. J. Gardiner and W. Hansen, The Riesz decomposition of finely superharmonic functions, Adv. Math. 214 (2007), 417-436.

[Ha] W. Hansen, Abbildungen harmonischer Räume mit Anwendung auf die Laplace und Wärmeleitungsgleichung, Ann. Inst. Fourier (Grenoble) 21 (1971), 203-216.

[HKM] J. Heinonen, T. Kilpeläinen, and O. Martio, Harmonic morphisms in nonlinear potential theory, Nagoya Math. J. 125 (1992), 115-140.

[Hs] M. Heins, On the Lindelöf principle, Ann. of Math. (2) 61 (1955), 440-473. 
[He] R.-M. Hervé, Recherches axiomatiques sur la théorie des fonctions surharmoniques et du potentiel, Ann. Inst. Fourier (Grenoble) 12 (1962), 415-571.

[Ish] T. Ishihara, A mapping of Riemannian manifolds which preserves harmonic functions, J. Math. Kyoto Univ. 19 (1979), 215-229.

[Jac] C. G. J. Jacobi, Über eine Lösung der partiellen Differentialgleichung $\frac{\partial^{2} V}{\partial x^{2}}+\frac{\partial^{2} V}{\partial y^{2}}+$ $\frac{\partial^{2} V}{\partial z^{2}}=0$, J. Reine Angew. Math. 36 (1848), 113-134.

[Jan] K. Janssen, A cofine domination principle for harmonic spaces, Math. Z. 141 (1975), 185-191.

[La1] I. Laine, Covering properties of harmonic Bl-mappings, II, Ann. Acad. Sci. Fenn. Math. 570 (1974), 3-13.

[La2] , Covering properties of harmonic Bl-mappings, III, Ann. Acad. Sci. Fenn. Math. 1 (1975), 309-325.

[Me] C. Meghea, Compactification des espaces harmoniques, Lecture Notes in Math. 222, Springer, Berlin, 1971.

Department of Mathematical Sciences

University of Copenhagen

2200 Copenhagen Ø

Denmark

fuglede@math.ku.dk 